\title{
Treatment with Combinations of the Anti-HER2 Antibody Trastuzumab and Capecitabine in Advanced Metastatic Breast Cancer Patients - a Pilot Study
}

\author{
Michael Braun ${ }^{a} \quad$ Bettina Schlehe $^{b} \quad$ Andree Faridic $^{c}$ \\ Uwe Heindrichs ${ }^{d}$ \\ a Department of Gynecology and Obstetrics, University Hospital Bonn, \\ ${ }^{b}$ Department of Gynecology and Obstetrics, University Hospital Heidelberg, \\ ' Department of Gynecology, Asklepios Klinik, Hamburg-Barmbek, \\ ${ }^{\mathrm{d}}$ Department of Breast Surgery, Department of Gynecology and Obstetrics, University Hospital Aachen, Germany
}

Christian Rudlowski

\section{Key Words}

HER2 - Capecitabine - Trastuzumab - Breast cancer, metastatic

\section{Summary}

Background: Metastatic HER2-overexpressing breast cancer patients mostly are intensively pretreated patients. Tumor progression requires an effective and well tolerated treatment. Here, we report of a study with capecitabine/trastuzumab combination therapy, with regard to tumor response and safety in advanced metastatic breast cancer overexpressing HER2. Patients and Methods: A cohort of 20 metastatic patients suffering from HER2-overexpressing carcinoma were treated with capecitabine/trastuzumab. The patients had progressive disease after taxane/trastuzumab combination therapy. All patients received a standard dose of capecitabine $1,250 \mathrm{mg} / \mathrm{m}^{2}$ twice daily, administered on days 1-14, combined with $2 \mathrm{mg} / \mathrm{kg}$ weekly trastuzumab. Trastuzumab treatment was continued from previous treatment regimens. Safety and tolerability of this regimen were evaluated. Results: Response rate and time to progression were documented. The median number of treatment cycles was 10 (range: 3-18). The median dose of capecitabine was $2,100 \mathrm{mg} / \mathrm{m}^{2}$ daily. 2 patients were reported to have complete response, 6 partial response, and 5 stable disease. 7 patients had progressive disease. Median time to progression was 30 weeks. The treatment was well tolerated with little side effects. Only palmar-plantar erythema and gastrointestinal disorders required dose reductions. Conclusion: Combination of capecitabine/trastuzumab is an effective and safe regimen in pretreated HER2-positive metastatic breast cancer. Our results comfirm preclinical observations of an at least additive effect of HER2 antibody treatment with capecitabine.

\author{
Schlüsselwörter \\ HER2 - Capecitabin · Trastuzumab - Mammakarzinom, \\ metastasiertes
}

\section{Zusammenfassung}

Hintergrund: Trotz intensiver Vortherapie kommt es bei Patientinnen mit metastasiertem, HER2-positivem Mammakarzinom oft zum Tumorprogress. In dieser Situation ist eine effektive und gut verträgliche zytotoxische Therapie erforderlich. Wir berichten von einer Studie mit der Therapiekombination Capecitabin/Trastuzumab für vorbehandelte Patientinnen mit HER2-positiven, metastasierten Mammakarzinomen. Patienten und Methoden: 20 Patientinnen wurden mit Capecitabin/Trastuzumab behandelt. Sie waren mit anthrazyklin- und/oder taxanhaltigen Regimen vorbehandelt und hatten Tumorprogress unter Trastuzumab-Therapie. Pro Zyklus wurden $1.250 \mathrm{mg} / \mathrm{m}^{2}$ Capecitabin zweimal täglich über 2 Wochen gegeben in Kombination mit $2 \mathrm{mg} / \mathrm{kg}$ Trastuzumab wöchentlich. Die Therapie wurde bis zum Progress fortgesetzt oder bei Auftreten von schwerwiegenden Nebenwirkungen abgesetzt. Verträglichkeit und Ansprechen wurden evaluiert. Ergebnisse: Die mediane Anzahl der applizierten Zyklen war 10 (Bereich: 3-18). Die mediane CapecitabinDosierung lag bei $2.100 \mathrm{mg} / \mathrm{m}^{2} / \mathrm{Tag}$. 2 Patientinnen zeigten eine komplette, 6 eine partielle klinische Remission, 5 eine Stabilisierung der Erkrankung. 7 Patientinnen zeigten eine primäre Progression unter Therapie. Die mediane progressionsfreie Zeit betrug 30 Wochen. Die Therapie war gut verträglich bei nur geringen Nebenwirkungen. Auftreten von palmoplantaren Erythemen und Magen-Darmbeschwerden erforderte eine Dosisreduktion. Schlussfolgerung: Capecitabin/Trastuzumab ist eine effektive und nebenwirkungsarme Therapie für vorbehandelte, HER2-positive Patientinnen mit metastasierten Mammakarzinomen. Das gute und vergleichsweise lang anhaltende Ansprechen dieser Therapiekombination bestätigt Untersuchungen, die zumindest einen additiven zytotoxischen Effekt der Therapiekombination nahelegen.

\begin{tabular}{ll}
\hline KARGER & @ 2007 S. Karger GmbH, Freiburg \\
Fax +49 7614520714 & Accessible online at: \\
$\begin{array}{l}\text { E-mail Information@Karger.de } \\
\text { www.karger.com }\end{array}$ & www.karger.com/brc \\
&
\end{tabular}

PD Dr. med. Christian Rudlowski

Zentrum für Geburtshilfe und Frauenheilkunde der Universität Bonn

Abteilung für Frauenheilkunde und gynäkologische Onkologie

Sigmund-Freud-Str.25, 53127 Bonn, Germany

Tel. +49 228287-15447, Fax -6092

E-mail rudlowski@t-online.de 


\section{Introduction}

Despite early diagnosis and new treatment options, a substantial part of breast cancer patients will develop metastatic disease that cannot be cured with conventional treatments [1] Therefore, an increasing number of patients presents with anthracycline- and taxane-pretreated metastatic breast cancer since these highly active agents are used earlier in the course of the disease, including adjuvant treatment. Until recently, there have been no standard treatment options for these patients [2]. New regimens are missing particularly for women with breast cancer that overexpresses HER2, being an aggressive form of the disease with significantly shortened diseasefree survival and overall survival [3]. With the introduction of the monoclonal antibody trastuzumab, either alone or in combination with chemotherapy, clinical benefit could be demonstrated in patients with metastatic breast cancer that overexpresses HER2 $[4,5]$. The addition of trastuzumab to an anthracycline- or taxane-containing chemotherapy is associated with longer time to disease progression, a higher rate of objective response and longer duration of response (overall survival benefit) [5]. Despite the longer survival time, only few patients with metastatic disease are cured, and treatment frequently causes a reduction of the performance status or severe adverse events. Tumor progression requires subsequent cytotoxic treatment which is not well defined. Besides an excellent cytotoxic effect, following treatment regimens should be safe and well tolerated.

Capecitabine is an effective oral chemotherapy for metastatic breast cancer, both as monotherapy and in combination with other cytotoxic drugs [6]. Capecitabine is a rationally designed, tumor-activated, oral fluoropyrimidine that mimics continuous infusion of 5-fluorouracil (5-FU). It is converted to 5-FU preferentially at the tumor site through exploitation of the high intratumoral concentrations of the enzyme thymidine phosphorylase [7]. Capecitabine is a promising and well tolerated first- and second-line monotherapy for women with metastatic breast cancer [8]. It has consistently shown high efficacy in patients who have taxane-pretreated metastatic breast cancer, achieving objective response rates of $18-29 \%$ and a survival duration of approximately 1 year [6]. Capecitabine demonstrates a favorable safety profile, comparable to continuous 5-FU infusion regimens. The predominant adverse events are cutaneous (hand-foot syndrome, palmoplantar erythrodysesthesia (PPE)), and gastrointestinal (nausea/vomiting, diarrhea, stomatitis). Adverse events are typically mild to moderate in intensity and can be controlled by treatment interruption and dose adjustment to the individually tolerated dose without loss of effectiveness [9]. There is no evidence of cumulative toxicity, and the incidence of grade 3-4 adverse events is generally low. Clinical grade 4 adverse events occur in $2 \%$ of patients [6].

Capecitabine is suitable for combination with a variety of other cytotoxic agents. The favorable safety profile, with a par-
Table 1. Inclusion and exclusion criteria of the study

Inclusion criteria $\quad$ Written informed consent
Histologically confirmed HER2-positive breast
cancer
Progressive metastatic breast cancer
Bidimensionally measurable metastatic tumor site
according to RECIST criteria
Pretreatment with either paclitaxel or docetaxel
and trastuzumab
Left ventricular ejection fraction within the
institution's normal range
Life expectancy of at least 12 weeks
ECOG performance status of $\leq 2$
Adequate hematic, renal and hepatic function
Adverse cardiac events during trastuzumab
treatment, cardiac output $<50 \%$
Known breast cancer metastatis of the central
nervous system

ticularly low rate of myelosuppression, and the oral application provide an excellent basis for combination with other effective and well tolerated agents for patients with metastatic disease in moderate or poor performance status. The combination with trastuzumab is an attractive therapeutic approach. Trastuzumab plus chemotherapy is considered to be standard therapy for patients with HER2-overexpressing tumors [5]. Since trastuzumab and capecitabine have distinct safety profiles, there is a clear rationale for investigating them in combination. Furthermore, capecitabine and trastuzumab have at least additive activity in a human breast cancer xenograft model that overexpresses HER2 and is sensitive to trastuzumab monotherapy [10]. Capecitabine/trastuzumab as combination therapy has been investigated in a pilot trial including anthracycline- and taxane-pretreated metastatic breast cancer patients who overexpress HER2 [11]. The favorable response rates in this subset of patients which is difficult to treat has provided the rationale for the clinical investigation of the combination.

In the study reported here, we treated metastatic breast cancer patients who failed combination therapy with taxane/ trastuzumab. The purpose of the present pilot phase II study was to evaluate tumor response rate and safety of the combination capecitabine/trastuzumab.

\section{Patients and Methods}

\section{Patients}

Patients with HER2-overexpressing metastatic breast cancer were enrolled in this study from June 2002 to September 2004. They had tumor progression after treatment with regimens that included an anthracycline, a taxane, and trastuzumab. They received capecitabine/trastuzumab treatment at the University Hospitals Heidelberg and Aachen, Germany. Inclusion criteria were defined as follows (table 1): patients with progressive, HER2-positive metastatic breast cancer; bidimensionally measurable 
Table 2. Baseline patient characteristics $(\mathrm{n}=20)$

Table 3. Prior treatment history of patients $(n=20)$

\begin{tabular}{ll}
\hline & Patients, $\mathrm{n}(\%)$ \\
\hline Chemotherapy setting & \\
Primary systemic & 3 \\
Adjuvant & 12 \\
Metastatic treatment & \\
First-line & 20 \\
Second-line & 10 \\
Third-line & 3 \\
Chemotherapeutic agents & \\
Anthracyclines & 20 \\
Taxanes & 20 \\
Infused 5-FU & 9 \\
Trastuzumab & \\
First-line & 12 \\
Second-line & 10 \\
Response to taxane/trastuzumab treatment & $11 / 20(55)$ \\
Complete & $4(20)$ \\
Partial & $7(35)$ \\
Stable disease & $3(15)$ \\
Progressive disease & $6(30)$ \\
\hline
\end{tabular}

metastatic tumor site according to the Response Evaluation Criteria in Solid Tumors (RECIST); first or second line treatment with either paclitaxel or docetaxel and trastuzumab; left ventricular ejection fraction (LVEF) within the institution's normal range; life expectancy of at least 12 weeks; adequate renal, hepatic, and hematologic function. Patients were excluded if the left cardiac ventricular output was $<50 \%$, or if they had known breast cancer metastasis of the central nervous system. The protocol was approved by the local ethics committee. The trial was conducted in accordance with the Declaration of Helsinki and its subsequent amendments. Expression of HER2 was determined by immunohistochemical analysis of tumor tissue, collected either at the time of primary diagnosis or at recurrence, using the HercepTest ${ }^{\mathrm{TM}}$ (Dako Corp., Hamburg, Germany). All patients enrolled had 3+ overexpression according to standard criteria.
Table 4. Number of treatment cycles, best response, duration of response, and dose intensity of capecitabine in the patients group

\begin{tabular}{lc}
\hline & Median (range) \\
\hline Patients, total $\mathrm{n}$ & 20 \\
Treatment cycles, $\mathrm{n}$ & $8(3-18)$ \\
Dose intensity of capecitabine, $\mathrm{mg} / \mathrm{m}^{2}$ & $2,100(1,400-2,500)$ \\
Time to progression, days & $30(0-56)$ \\
Response, patients, $\mathrm{n}$ & \\
Complete & 2 \\
Partial & 6 \\
Stable disease & 5 \\
Progressive disease & 7 \\
\hline
\end{tabular}

\section{Treatment}

All patients received the standard dose of oral capecitabine of 1,250 $\mathrm{mg} / \mathrm{m}^{2}$ twice daily, administered on days $1-14$, followed by a 7 -day rest period. Dose reduction and modification was carried out according to the severity of the adverse event $[12,13]$. The start of a new treatment cycle was delayed until treatment-related adverse events resolved to at least mild intensity. Trastuzumab was administered intravenously on an outpatient basis at a weekly dose of $2 \mathrm{mg} / \mathrm{kg}$. Informed consent was obtained from all patients enrolled in this study.

\section{Patient Assessment}

Tumor response was evaluated by an independent physician before and after every 3 rd cycle of capecitabine using standard clinical and/or radiological methods, and the best response achieved by each patient was recorded. Efficacy was classified as progressive disease (PD), stable disease (SD), partial response (PR), or complete response (CR). Clinical assessments were performed at base line, at least every 3 cycles of capecitabine, and at the time the patient's treatment was discontinued. Abnormalities in laboratory values were classified by the grading system of the World Health Organization, and cardiac dysfunction by the criteria of the New York Heart Association.

Adverse events were graded based on their severity: grade 1 (mild), grade 2 (moderate), grade 3 (severe), or grade 4 (life-threatening), according to the National Cancer Institute Common Toxicity Criteria (NCI CTC) (version 2.0). Upper limits for laboratory values were as follows: neutrophils $1.5 \times 10^{9} / \mathrm{l}$, platelets $75 \times 10^{9} / 1$, hemoglobin $9 \mathrm{~g} / \mathrm{dl}$, serum bilirubin $2.0 \times$ upper limit of normal, and aspartate aminotransferase (AST), alanine aminotransferase (ALT) and alkaline phosphatase $2.5 \times$ upper limit of normal. PPE was graded 1-3 according to NCI CTC, which was also used in all clinical trials with capecitabine [12]. The incidence of treatment interruption and dose reduction was also recorded for all patients. In patients experiencing grade 2 or more severe toxicities, the standard capecitabine dose modification scheme, described in detail by Blum et al. [12], was applied. At the first occurrence of a grade 2 toxicity, treatment was interrupted and then resumed after resolution to grade 1 or better at the same dose. Subsequent occurrences of the same grade 2 toxicity were managed by treatment interruption followed by a $25 \%$ dose reduction. If grade 3 toxicity occurred, treatment was interrupted and followed by a 25 or $50 \%$ dose reduction. At the third occurrence of a given toxicity (grade 3 severity), treatment was discontinued, and the patient was withdrawn from the study. No dose reduction or interruption was required for anemia. Diarrhea, nausea, and vomiting were treated symptomatically.

\section{Evaluation Criteria}

The primary study end point was the overall response rate as measured by the number of CR and PR. Secondary end points included progressionfree survival (PFS), overall survival, and safety. A physical examination, 
including a neurological examination and complete blood count, was performed before the first treatment cycle. Pretreatment evaluation also included biochemical analyses, chest X-ray, and computed tomography (CT) scans to define the extent of the disease. Complete blood cell counts with differential and serum biochemistry analyses were repeated at each treatment cycle. Response was assessed radiologically every 3 cycles or when progression was suspected. Evaluations were performed by physical examination, chest X-ray, abdominal CT scan, or ultrasonography. CR, $\mathrm{PR}, \mathrm{SD}$, and PD were defined according to RECIST criteria.

\section{Statistical Analysis}

The trial was designed using the following testing procedure: Assuming a response rate of $\geq 10 \%, 22$ patients were to be included. The overall response rate (with $95 \%$ confidence interval (CI)) was calculated for all patients according to an intention-to-treat analysis. PFS was calculated from the first day of chemotherapy until the date of disease progression. OS was calculated from the start of study treatment until death. PFS curves were generated using the Kaplan-Meier method. Response duration was calculated from the date of response confirmation to the date of disease progression.

\section{Results}

\section{Patient Characteristics}

Because an ongoing phase III multicenter trial 'Treatment Beyond Progression' (TBP) of the same regimen in an identical patient population was started before this trial was completed, only 20 of the planned 22 patients were enrolled. The baseline characteristics of the patient cohort are shown in table 2. All of the 20 patients were evaluable for safety and tumor response. The median observation time was 44 (weeks, range: 12-58 weeks). The patients had visceral metastasis with at least 1 metastatic site. A significant proportion ( $8 / 20$ patients) had poor performance status (ECOG 2 or 3 ). All of the patients were anthracycline-pretreated in the adjuvant or metastatic setting. 7 patients had received 2,8 patients 3 , and 5 patients 4 prior cytotoxic regimens (table 3 ). All patients received the combination of trastuzumab and paclitaxel as firstor second-line treatment in the metastatic setting and showed tumor response in 14 of 20 cases (table 3 ). In this setting, the retrospective analysis revealed 4 patients $(20 \%)$ with CR, 7 patients $(35.0 \%)$ with PR, and 3 patients $(15 \%)$ with a SD.

\section{Efficacy}

At least 3 cycles of capecitabine in combination with trastuzumab were administered. 2 patients had a CR, and 6 patients a PR. Additionally, there were 5 patients with SD, and 7 patients with PD (table 4). Of the 2 patients with CR, 1 had pulmonary, mediastinal and hepatic disease, the other had hepatic, mediastinal and soft tissue disease. Tumor response could be achieved after only 3 cycles of therapy. Notably, tumor response was achieved in 7 patients who had previously received treatment with 5-FU in the adjuvant setting. The response to therapy was not associated with the hormone receptor status. It was remarkable that $3 / 6$ patients who did not respond to prior treatment achieved PR within this study.

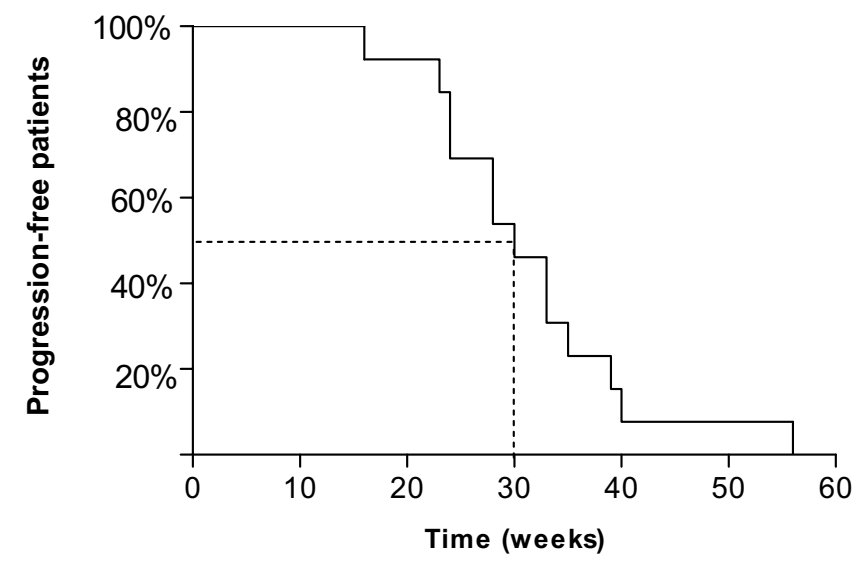

Fig. 1. Time to disease progression in the study group.

The median time to progression (TTP) was 30 weeks (range: 0-56 weeks; fig. 1 ). For the 2 patients with a CR, it was 38 and 56 weeks, respectively. Treatment was interrupted in 7 patients who were non-responders concerning initial therapy with trastuzumab/capecitabine. In 11 patients, treatment was stopped due to PD after initial response to therapy. If indicated, patients were treated with chemotherapy for PD and received a variety of different chemotherapy regimens including vinorelbine, gemcitabine and liposomal doxorubicine. Median OS of the study group was 53 weeks (range: 12-106 weeks). In all of the patients, a disease-related death was documented.

\section{Safety}

In total, 167 treatment cycles were administered, with each patient receiving a median of 8 cycles (range: $3-18$ ). The median dose of capecitabine was $2,100 \mathrm{mg} / \mathrm{m}^{2}$ daily (range: 1,400-2,500 mg). Cycles 1-3 were administered without dose reduction or delay of more than 1 week for all of the patients. In addition, within the first 6 cycles, no interruption was required, therapy-related adverse events were rare and mild to moderate in intensity. In patients receiving more than 6 cycles (11/20), treatment delay (less than 2 weeks) and dose reductions were common. Trastuzumab was administered without dose modification or delay. The most common treatment-related adverse events (fig. 2) were PPE (9/20), gastrointestinal side effects $(5 / 20)$, and nausea (5/20). Treatment-related hematologic adverse events were rare, and no grade $3 / 4$ events occurred. No grade 3/4 alopecia was observed. There were no grade $3 / 4$ alterations in laboratory parameters (table 5).

Dose reduction due to adverse events was required in 9/20 patients, and the adverse events that most commonly lead to a capecitabine dose modification (delay, reduction or interruption) were PPE (6 patients), diarrhea (3 patient), and stomatitis/mucositis (4 patients). In none of the patients, adverse cardiac events were reported during or at the end of treatment. 
Fig. 2. Most common ( $>10 \%$ of patients) treatment-related adverse events classified according to the National Cancer Institute Common Toxicity Criteria (NCI CTC) (version 2.0). Palmo-plantar erythrodysesthesia (PPE) was graded 1-3 according to NCI CTC, which was also used in all clinical trials with capecitabine.

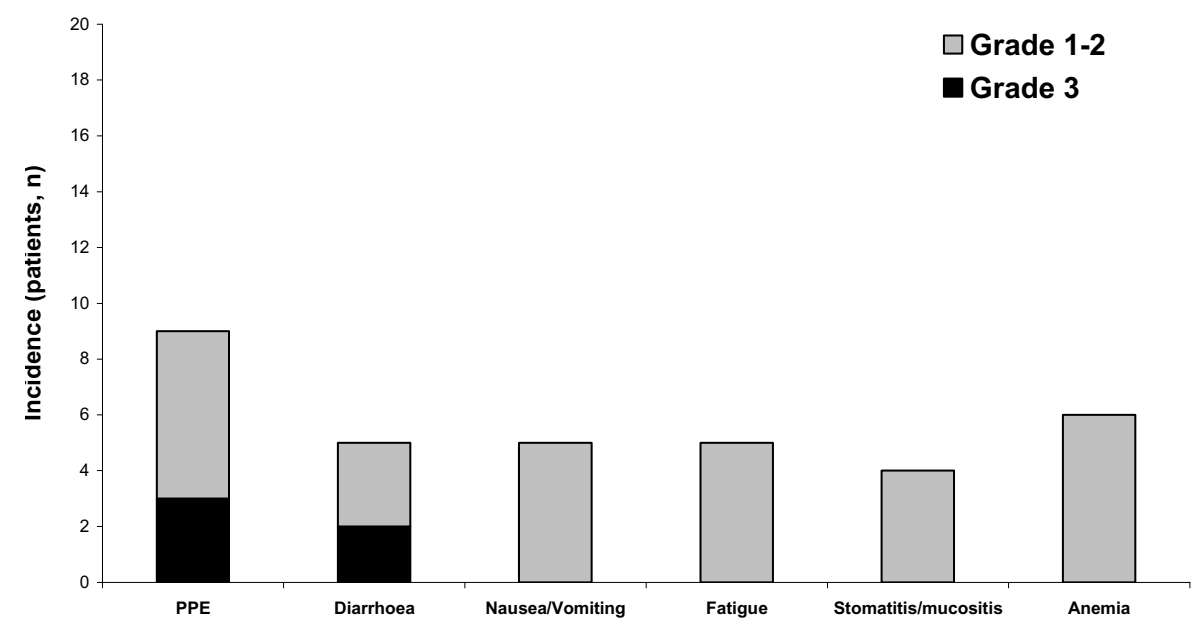

In 2 cases with $\mathrm{CR}$, treatment had to be interrupted after 11 and 18 cycles as a result of PPEs and gastrointestinal disorders. In these patients, the starting dose was reduced (at least twice daily $1,000 \mathrm{mg} / \mathrm{m}^{2}$ ).

\section{Discussion}

Metastatic HER2-positive breast cancer shows a more aggressive course of the disease that is known to be less sensitive to cytotoxic treatment compared to HER2-negative disease. Both limited response rate and duration of response are known to be characteristic for those tumors [14]. The combination therapy of trastuzumab and taxanes improves the response rates and OS significantly and is considered as standard treatment in first-line therapy [4]. Nevertheless, the inevitable disease progression requires subsequent treatment. A number of chemotherapeutic approaches have been tested in patients failing taxane treatment, and most data show capecitabine to be the superior treatment [2]. However, no optimal treatment has been established in this setting for a combination with trastuzumab. Currently, no data are available that show superiority of a combination of trastuzumab/chemotherapy compared to chemotherapy alone after initial treatment with trastuzumab/taxane regimens [15].

The trial described in this report was undertaken to provide trastuzumab therapy for patients whose metastatic breast cancer progressed during treatment with taxane/trastuzumab. Our study shows that the treatment with capecitabine/ trastuzumab in patients with taxane-pretreated HER2-positive metastatic breast cancer is save and effective. This is due to a high response rate of $8 / 20$ and additional disease stabilization in 5/20 patients associated with a favorable and manageable safety profile especially in patients with moderate or poor performance status $(8 / 20 \mathrm{ECOG} \geq 2)$. This cytotoxic efficacy could be demonstrated although all of the patients were pretreated with taxanes in combination with trastuzumab. In our study group, pretreatment with taxane/trastuzumab combination was highly effective (objective response 55.0\%, TTP 30 weeks) which is in line with the literature [5]. Interestingly, response to therapy was shown in particular for visceral tumor sites, and response duration was prolonged. Nevertheless, it is unknown if this efficacy is a result of synergistic or additive cytotoxic mechanisms. Our results are consistent with preliminary findings from a small phase II study showing encouraging clinical response rates for capecitabine/trastuzumab in extensively pretreated patients with advanced breast cancer [11] They are not in line with data from cell culture experiments [16]. These in vitro analyses showed an antagonism of trastuzumab and 5-FU in SK-BR-3 cell lines. In the same study, other cytotoxic agents like paclitaxel or cisplatin demonstrated a synergistic effect in combination with trastuzumab. It was supposed that trastuzumab is responsible for a reduction of tumor cells passing through the S-phase fraction of the cell cycle. Accordingly, the efficacy of 5-FU would diminish, since its cytotoxicity is based on the inhibition of the DNA/RNA synthesis. These findings could not be confirmed in an animal model. In experiments with a human breast cancer xenograft model, in vivo antitumor activity of the combination capecitabine/trastuzumab was at least additive in terms of tumor growth inhibition and tumor growth delay [10]. Any antagonistic interaction found in vitro was not observed in vivo. It is likely that the mechanism of the antitumor activity of trastuzumab in vivo is different from its in vitro activity in the tumor models studied. Indeed, mechanisms like angiogenesis, antibody-dependent cell-mediated cytotoxicity (ADCC) and cell-matrix interactions have recently been shown to be important for the in vivo activity of trastuzumab [17]. These actions are independent from the cytotoxic mechanisms in which 5-FU is involved. However, administration of trastuzumab did not affect the tumor levels of the thymidine phosphorylase like it was shown for paclitaxel [10].

Several studies have analyzed the safety and toxicity profile of capecitabine as monotherapy for metastatic breast cancer [18]. 
Table 5. Summary of treatment-related adverse events (occurring in $\geq 10 \%$ of patients)

\begin{tabular}{|c|c|c|c|c|}
\hline \multirow[t]{2}{*}{ Location } & \multicolumn{4}{|l|}{ Patients, $\mathrm{n}$} \\
\hline & Grade $1^{\mathrm{a}}$ & Grade $2^{\mathrm{a}}$ & Grade $3^{\mathrm{a}}$ & Grade $4^{\mathrm{a}}$ \\
\hline \multicolumn{5}{|l|}{ Skin } \\
\hline $\mathrm{PPE}^{\mathrm{b}}$ & 2 & 4 & 2 & - \\
\hline \multicolumn{5}{|l|}{ Gastrointestinal system } \\
\hline Nausea/vomiting & 3 & 2 & - & - \\
\hline Diarrhea & - & 2 & 3 & - \\
\hline Stomatitis/mucositis & 2 & 2 & - & - \\
\hline Anorexia & 2 & 1 & - & - \\
\hline \multicolumn{5}{|l|}{ Liver/biliary system } \\
\hline GOT/GPT & 2 & 2 & - & - \\
\hline Bilirubin increased & 3 & - & - & - \\
\hline \multicolumn{5}{|l|}{ Metabolic/nutritional system } \\
\hline Raised alkaline phosphatase & 2 & 1 & - & - \\
\hline Increased LDH & 1 & - & - & - \\
\hline \multicolumn{5}{|l|}{ Blood } \\
\hline Anemia $^{c}$ & 2 & 4 & - & - \\
\hline Neutropenia & - & 1 & - & - \\
\hline \multicolumn{5}{|l|}{ General } \\
\hline Fever & - & - & - & - \\
\hline Fatigue & 3 & 2 & - & - \\
\hline Alopecia & - & 2 & - & - \\
\hline
\end{tabular}

PPE $=$ Palmo-plantar erythrodysesthesia $;$ GOT $=$ glutamic oxaloacetic transaminase; $\mathrm{GPT}=$ glutamic pyruvic transaminase $\mathrm{LDH}=$ lactate dehydrogenase .

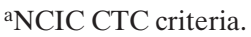

bPPE was graded 1-3 according to NCI-CTC also used in all clinical trials with capecitabine.

${ }^{\mathrm{c} T o t a l}$ of decreased red blood cell count and decreased hemoglobin.
To summarize the results, side effects were low and treatment was well tolerated. PPEs and gastrointestinal disorders were the leading symptoms that required dose modifications and treatment interruption. In our study, the toxicity and safety profiles were comparable to data available from studies with capecitabine only. No remarkable side effects could be attributed to trastuzumab in particular.

\section{Conclusion}

Despite potential tumorbiological discrepancies between in vitro and in vivo effects, our study shows that the combination capecitabine/trastuzumab could be a new, convenient and tumor-specific treatment approach in the HER2-positive metastatic situation. It is easy to administer, well tolerated, and accepted by all treated patients. Its use provides patients with a degree of independence potentially improving quality of life. Our results warrant further trials to optimize the treatment in this subset of patients. Therefore, data from the TBP study conducted by the German Breast Group are eagerly awaited.

\section{Acknowledgement}

The authors would like to thank Dr. Astrid Kiermair and Dr. Regina Moka for editing the manuscript.

\section{References}

1 Hortobagyi GN, Piccart-Gebhart MJ: Current management of advanced breast cancer. Semin Oncol 1996;23:1-5

2 Seidman AD, O'Shaughnessy J, Misset JL: Singleagent capecitabine: a reference treatment for taxane-pretreated metastatic breast cancer? Oncologist 2002;7(suppl 6):20-28.

3 Slamon DJ, Clark GM, Wong SG, Levin WJ, Ulrich A, McGuire WL: Human breast cancer: correlation of relapse and survival with amplification of the HER-2/neu oncogene. Science 1987;235:177-182.
4 Cobleigh MA, Vogel CL, Tripathy D, Robert, NJ, Scholl S, Fehrenbacher L, Wolter JM, Paton V, Shak S, Lieberman G, Slamon DJ: Multinational study of the efficacy and safety of humanized antiHER2 monoclonal antibody in women who have HER2-overexpressing metastatic breast cancer that has progressed after chemotherapy for metastatic disease. J Clin Oncol 1999;17:2639-2648.
Slamon DJ, Leyland-Jones B, Shak S, Fuchs H, Paton V, Bajamonde A, Fleming T, Eiermann W, Wolter J, Pegram M, Baselga J, Norton L: Use of chemotherapy plus a monoclonal antibody against HER2 for metastatic breast cancer that overexpresses HER2. N Engl J Med 2001;344:783-792.

6 Wagstaff AJ, Ibbotson T, Goa KL: Capecitabine: a review of its pharmacology and therapeutic efficacy in the management of advanced breast cancer. Drugs 2003;63:217-236. 
7 Miwa M, Ura M, Nishida M, Sawada, N, Ishikawa T, Mori K, Shimma N, Umeda I, Ishitsuka H: Design of a novel oral fluoropyrimidine carbamate, capecitabine, which generates 5-fluorouracil selectively in tumours by enzymes concentrated in human liver and cancer tissue. Eur J Cancer 1998; 34:1274-1281.

8 O'Shaughnessy J: Clinical experience of capecitabine in metastatic breast cancer. Eur J Cancer 2002;38(suppl 2):10-14.

9 O'Shaughnessy J, Twelves C, Aapro M: Treatment for anthracycline-pretreated metastatic breast cancer. Oncologist 2002;7(suppl 6):4-12.

10 Fujimoto-Ouchi K, Sekiguchi F, Tanaka Y: Antitumor activity of combinations of anti-HER-2 antibody trastuzumab and oral fluoropyrimidines capecitabine/5'-dFUrd in human breast cancer models. Cancer Chemother Pharmacol 2002;49: 211-216.

11 Bangemannn N: Treatment of HER2 overexpressing metastatic breast cancer (MBC) with trastuzumab (Herceptin) and chemotherapy. Breast Cancer Res Treat 2000;64:123.

-12 Blum JL, Jones SE, Buzdar AU, LoRusso PM; Kuter I, Vogel C, Osterwalder B, Burger HU, Brown CS, Griffin T: Multicenter phase II study of capecitabine in paclitaxel-refractory metastatic breast cancer. J Clin Oncol 1999;17:485-493.

13 Cassidy J, Twelves C, Van Cutsem E, Hoff P, Bajetta E, Boyer M, Bugat R, Burger U, Garin A, Graeven U, McKendric J, Maroun J, Marshall J, Osterwalder B, Perez-Manga G, Rosso R, Rougier P, Schilsky RL: First-line oral capecitabine therapy in metastatic colorectal cancer: a favorable safety profile compared with intravenous 5-fluorouracil/leucovorin. Ann Oncol 2002;13:566-575.

14 Pegram MD, Pauletti G, Slamon DJ: HER-2/neu as a predictive marker of response to breast cancer therapy. Breast Cancer Res Treat 1998;52:65-77.

15 Bell R, Verma S, Untch M, Cameron D, Smith I Maximizing clinical benefit with trastuzumab. Semin Oncol 2004;31:35-44.

16 Pegram M, Hsu S, Lewis G, Pietras R, Beryt M, Sliwkowski M, Coombs D, Baly D, Kabbinavar F, Slamon D: Inhibitory effects of combinations of HER2/neu antibody and chemotherapeutic agents used for treatment of human breast cancers. Oncogene 1999;18:2241-2251.

17 Clynes RA, Towers TL, Presta LG: Inhibitory Fc receptors modulate in vivo cytoxicity against tumor targets. Nat Med 2000;6:443-446.

18 Ershler WB: Capecitabine monotherapy: safe and effective treatment for metastatic breast cancer. Oncologist 2006;11:325-335. 